\title{
Cardio- Renal Outcomes With Long- Term Agalsidase Alfa Enzyme Replacement Therapy: A I0- Year Fabry Outcome Survey (FOS) Analysis
}

This article was published in the following Dove Press journal: Drug Design, Development and Therapy

\begin{abstract}
Uma Ramaswami'
Michael Beck ${ }^{2}$

Derralynn Hughes'

Christoph Kampmann ${ }^{2}$

Jaco Botha ${ }^{3}$

Guillem Pintos-Morell (iD) ${ }^{4}$

Michael L West (D) $^{5}$

Dau-Ming Niu ${ }^{6}$

Kathy Nicholls iD ${ }^{7,8}$

Roberto Giugliani (iD)

On behalf of the FOS Study Group

'Royal Free London NHS Foundation Trust, Lysosomal Disorders Unit, Institute of Immunity and Transplantation, London, UK;

${ }^{2}$ Centre for Paediatric and Adolescent Medicine, University Medical Centre, University of Mainz, Mainz, Germany; ${ }^{3}$ Department of Biostatistics and Programming, Takeda, Zug, Switzerland; ${ }^{4}$ Centre for Rare Diseases, University Hospital Vall d'Hebron, Barcelona, CIBERER-GCV08_IGTP Research Institute and Teaching Unit Germans Trias i Pujol, Universitat Autònoma de Barcelona, Badalona, Spain; ${ }^{5}$ Department of Medicine, Dalhousie University, Halifax, Nova Scotia, Canada; ${ }^{6}$ Department of Paediatrics, Taipei Veterans General Hospital, Taipei, Taiwan;

${ }^{7}$ Department of Nephrology, Royal Melbourne Hospital, University of Melbourne, Parkville, VIC, Australia; ${ }^{8}$ Department of Medicine, Royal Melbourne Hospital, University of Melbourne, Parkville, VIC, Australia; ${ }^{9}$ Medical Genetics Service HCPA, Department of Genetics, UFRGS, and INAGEMP, Porto Alegre, Brazil
\end{abstract}

Correspondence: Uma Ramaswami Lysosomal Disorders Unit, Institute of Immunity and Transplantation, 2nd Floor, Royal Free Hospital, Pond Street, London NW3 2QG, UK

Tel +44 2077940500 Ext 22492

Fax +442073177665

Email uma.ramaswami@nhs.net
Purpose: Following the publication of 5-year agalsidase alfa enzyme replacement therapy (ERT) outcomes data from the Fabry Outcome Survey (FOS), 10-year data were analyzed. Patients and methods: FOS (ClinicalTrials.gov identifier: NCT03289065) data (April 2001 to August 2018) were retrospectively analyzed. Estimated glomerular filtration rate (eGFR) and left ventricular mass indexed to height (LVMI) were analyzed after treatment start (baseline) for patients with $\geq 3$ measurements, including baseline and year 10 .

Results: Median (range) age (years) of the evaluable treated renal cohort at treatment start was $48.8(17.9-67.3)$ for females $(n=62), 34.4(18.0-66.8)$ for males $(n=90)$. With eGFR $\geq 60$ $\mathrm{mL} / \mathrm{min} / 1.73 \mathrm{~m}^{2}$ at baseline, mean $(95 \% \mathrm{CI})$ rate of eGFR change (eGFR/year) over 10 years was relatively stable in females $(\mathrm{n}=52 ;-0.55[-1.12,+0.01])$ and slightly declined in males $(\mathrm{n}=79 ;-1.99[-2.45,-1.54])$. With impaired kidney function $\left(\mathrm{eGFR}<60 \mathrm{~mL} / \mathrm{min} / 1.73 \mathrm{~m}^{2}\right)$ at baseline, mean $(95 \% \mathrm{CI})$ eGFR/year was stable in females $(\mathrm{n}=10 ;-0.14[-1.43,+1.15])$ and slightly declined in males $(\mathrm{n}=11 ;-2.79[-4.01,-1.56])$ over 10 years. Median (range) age (years) of the evaluable treated cardiac cohort at treatment start was 46.7 (3.7-67.3) for females $(n=34), 28.2(4.0-54.2)$ for males $(n=35)$. With left ventricular hypertrophy (LVH; LVMI $>48 \mathrm{~g} / \mathrm{m}^{2.7}$ in females, $>50 \mathrm{~g} / \mathrm{m}^{2.7}$ in males) at baseline, mean (95\% CI) LVMI/year slightly increased over 10 years in females $(n=18 ;+1.51[+0.91,+2.12])$ and males $(n=14$; $+0.87(+0.19,+1.55)$. Without LVH at baseline, mean $(95 \% \mathrm{CI}) \mathrm{LVMI} /$ year was stable in females $(n=16 ;+0.52[-0.13,+1.17])$ and males $(n=21 ;+0.57[+0.02,+1.13])$ over 10 years. Conclusion: Agalsidase alfa-treated patients with 10-year FOS data and preserved kidney function and/or normal LVMI at baseline remained largely stable; those with decreased kidney function or LVH at baseline experienced modest declines in renal function and/or increases in LVMI.

Keywords: agalsidase alfa, enzyme replacement therapy, Fabry disease, cardio-renal outcomes

\section{Introduction}

Fabry disease is a rare X-linked disorder caused by the lack of the lysosomal enzyme $\alpha$-galactosidase A, which affects glycosphingolipid metabolism. Consequences of this progressive disease include neuropathic pain, angiokeratoma, progressive renal damage leading to kidney failure, cardiomyopathy, arrhythmia, transient ischemic attacks and/or strokes, and reduced lifespan. ${ }^{1}$ The advent of enzyme replacement therapy (ERT) in 2001 offered the potential for improved outcomes in patients with Fabry disease as well as improving pain scores and measures of patient quality of 
life. ${ }^{2}$ Evidence suggests that improved outcomes may be particularly true when treatment is initiated early, before irreversible damage has occurred in target organs such as the kidney and heart. ${ }^{3-7}$ Fabry-specific therapy is used together with ancillary therapies for patients with renal or cardiac disease, such as angiotensin receptor blockers, angiotensin-converting enzyme inhibitors, and interventions aimed at arrhythmia and congestive heart failure. ${ }^{7}$

The Fabry Outcome Survey (FOS), initiated by Shire (now part of Takeda) in 2001, collects long-term data from patients in 24 countries on the natural history of Fabry disease and the safety and efficacy of treatment with agalsidase alfa. ${ }^{8}$ A previous evaluation of FOS data from patients who had received 5 years of agalsidase alfa ERT showed this was associated with reduced morbidity and mortality. ${ }^{9}$ The current objective was to perform an additional retrospective analysis of data extracted from FOS in August 2018 to assess cardiac and renal outcomes in patients who had received agalsidase alfa ERT over a 10year period.

\section{Materials And Methods Patients}

The FOS all treated 10-year cohort $(n=388)$ comprised all children and adults followed in FOS who initiated agalsidase alfa ERT $0.2 \mathrm{mg} / \mathrm{kg}$ intravenously every other week either before or during entry into FOS and received it for a minimum of 10 years before data extraction on August 3, 2018. Of 350 patients ( $n=38$ missing) in the all-treated cohort, we identified that 197 (56.3\%) patients within the cohort were carrying mutations related to classic Fabry disease and $35(10.0 \%)$ patients were carrying mutations related to late-onset Fabry disease; 118 (33.7\%) patients were of unknown phenotype.

The FOS evaluable treated cohort $(n=363)$ was defined as the subset of patients who did not receive other ERT prior to FOS entry, had a known agalsidase alfa ERT start date and did not have a kidney transplant or receive dialysis before the start of treatment; this cohort included 44 pediatric ( $<18$ years of age) and 319 adult $(\geq 18$ years of age) patients at FOS entry, and 53 pediatric and 310 adult patients at ERT start. Patients were excluded if they received other ERT before FOS entry $(n=11)$ or had begun dialysis or had a kidney transplant prior to ERT initiation ( $\mathrm{n}=14$; Figure 1).

The FOS evaluable treated renal cohort $(n=152$; all adults except 1 pediatric patient at FOS entry but no pediatric patients at FOS extract) comprised patients from the evaluable treated cohort with complete longitudinal data, i.e. those who had $\geq 3$ available estimated glomerular filtration rate (eGFR) measurements after ERT start, including the baseline and year 10 time points (Figure 1). The FOS evaluable treated cardiac cohort $(n=69$; all adults except 10 pediatric patients at FOS entry and 1 pediatric patient at FOS extract) included patients from the evaluable treated cohort with complete longitudinal data, i.e. patients with $\geq 3$ available left ventricular mass indexed to height (LVMI) measurements after ERT start, including the baseline and year 10 time points (Figure 1).

Of the patients excluded from the evaluable treated cohorts, 3 patients were excluded from the renal and cardiac cohorts due to switching to another treatment (type not specified; as entered by the investigator as free text) before their 10-year data could be collected.

FOS (ClinicalTrials.gov identifier: NCT03289065) has been approved by the ethics institutional review boards of participating centers (Supplemental File). Further, this registry was compliant with relevant global and local regulations and best practices. Good Pharmacoepidemiological Practice, Good Research for Comparative Effectiveness principles, and the relevant principles of the International Conference on Harmonisation Good Clinical Practice (ICH GCP) guidelines (ICH E6) were followed as appropriate for an observational registry. All participants gave written informed consent.

\section{Assessments}

eGFR was calculated only in patients aged $\geq 18$ years at the time of assessment, using serum creatinine level and an equation derived from the Modification of Diet in Renal Disease study. ${ }^{10}$ LVMI was calculated using the Devereux formula from measurements obtained by echocardiography. ${ }^{11}$

Left ventricular hypertrophy (LVH) was defined as LVMI $>48 \mathrm{~g} / \mathrm{m}^{2.7}$ in females and LVMI $>50 \mathrm{~g} / \mathrm{m}^{2.7}$ in males. ${ }^{12,13}$ LVMI slopes over 10 years were analyzed by sex and baseline LVH status, and left ventricular posterior wall thickness in diastole (PWTD) slopes over 10 years were analyzed by sex and baseline eGFR $<60$ or $\geq 60 \mathrm{~mL} / \mathrm{min} / 1.73 \mathrm{~m}^{2}$.

\section{Statistical Analysis}

The statistical analysis was performed using $\mathrm{SAS}^{\circledR}$ software version 9.3 (SAS Institute Inc., Cary, NC, USA). Descriptive statistics were calculated for demographic and outcome data. For continuous variables, 95\% 


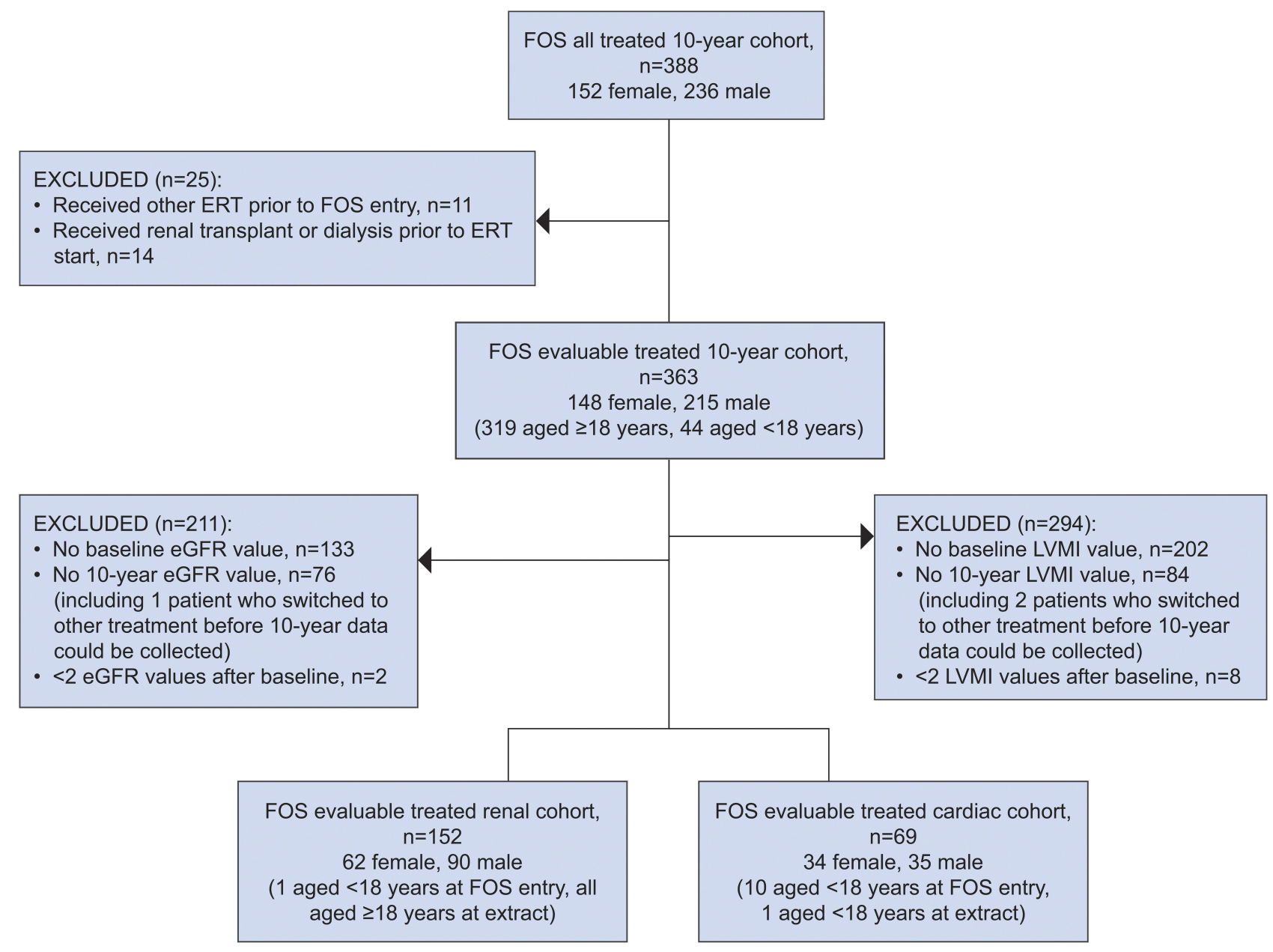

Figure I Flow diagram of participants included in the evaluable treated renal cohort and the evaluable treated cardiac cohort.

Abbreviations: eGFR, estimated glomerular filtration rate; ERT, enzyme replacement therapy; FOS, Fabry Outcome Survey; LVMI, left ventricular mass index.

confidence intervals (CIs) for means were computed using $t$-distribution. Average slope estimates for renal and cardiac parameters, together with standard errors (SEs) and 95\% CIs, were calculated using a mixed model with random intercept and slope, without any assumptions on the within-patient covariance structure (unstructured covariance matrix).

Mixed models for eGFR and LVMI included time from treatment start as covariate, sex and baseline renal (eGFR $<60$ or $\geq 60 \mathrm{~mL} / \mathrm{min} / 1.73 \mathrm{~m}^{2}$ ) or cardiac (LVH or no LVH) status as factors, and all corresponding interactions. Mixed model for PWTD included time from treatment start, sex, baseline eGFR level ( $<60$ or $\left.\geq 60 \mathrm{~mL} / \mathrm{min} / 1.73 \mathrm{~m}^{2}\right)$, and all interactions. In evaluable treated cardiac cohort patients with baseline eGFR $>90 \mathrm{~mL} / \mathrm{min} / 1.73 \mathrm{~m}^{2}$ and urinary protein $<0.5 \mathrm{~g} /$ day, the model for eGFR included time from treatment start as covariate, sex as a factor, and interaction of sex with time variable.

\section{Results}

\section{Evaluable Treated Renal Cohort}

The evaluable treated renal cohort consisted of 152 patients: 62 females with a median (range) age at treatment start of 48.8 (17.9-67.3) years and 90 males with a median (range) age at treatment start of 34.4 (18.0-66.8) years (Table 1). The median (range) time from treatment start to data extraction from FOS was 12.5 (10.1-17.1) years in females and $14.4(10.1-18.4)$ years in males (Table 1). Use of angiotensin-converting enzyme inhibitors and/or angiotensin receptor blockers was recorded in 41 of $62(66.1 \%)$ females and 73 of $87(83.9 \%)$ males (Table 1). Decreased kidney function (stage 3 or 4 chronic kidney disease, as patients on dialysis or having received a renal transplant were excluded) at baseline, defined as eGFR $<60 \mathrm{~mL} / \mathrm{min} / 1.73 \mathrm{~m}^{2}$, was seen in 10 of 62 (16.1\%) females and 11 of $90(12.2 \%)$ males. Baseline urinary protein data were available for 115 of $152(76 \%)$ 


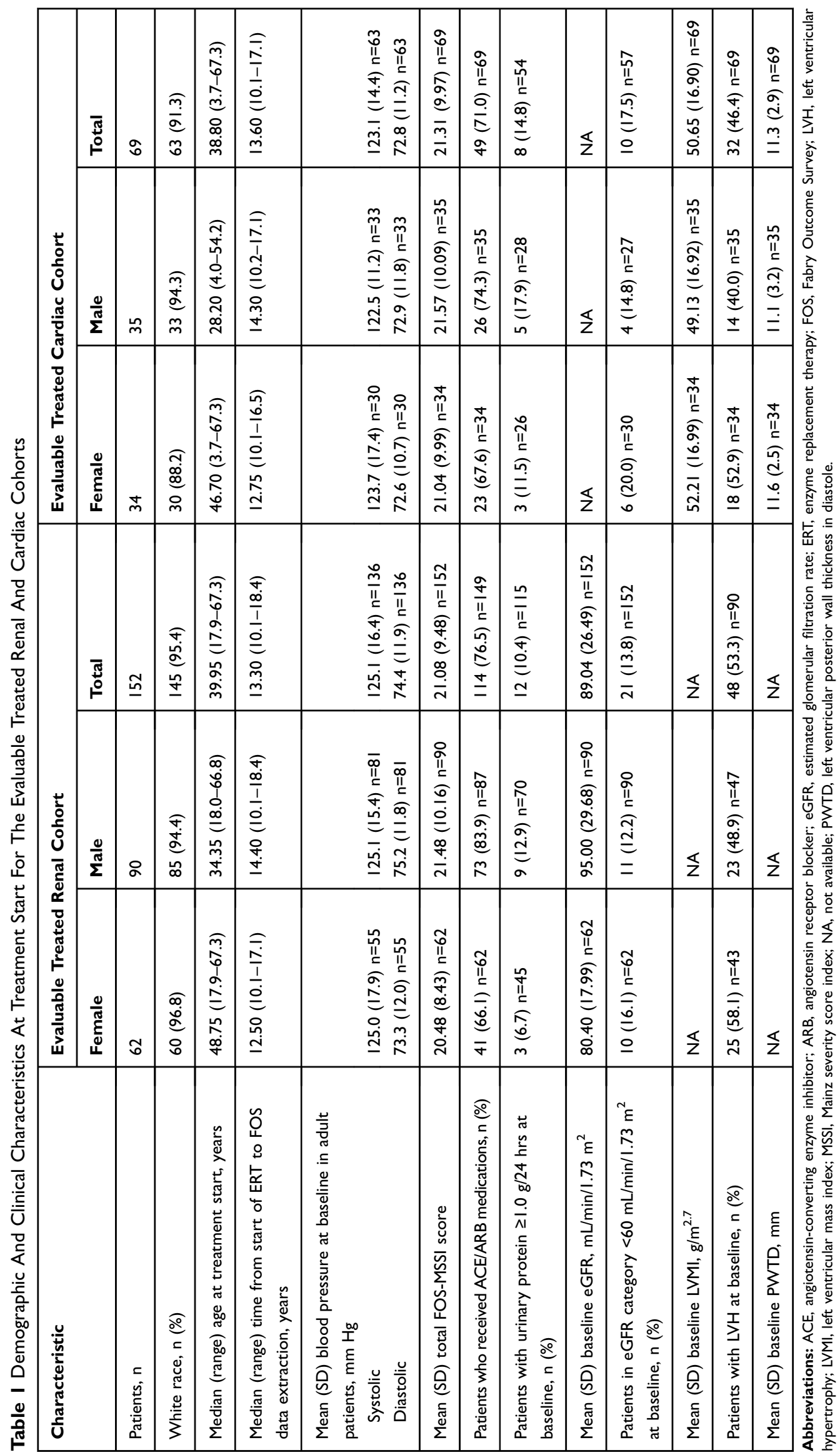


patients in the evaluable treated renal cohort and indicated urinary protein $\geq 1.0 \mathrm{~g} / 24 \mathrm{hrs}$ was present in 3 of $45(6.7 \%)$ females and 9 of 70 (12.9\%) males (Table 1).

The mean $(95 \% \mathrm{CI})$ eGFR measurements $(\mathrm{mL} / \mathrm{min} / 1.73$ $\mathrm{m}^{2}$ ) at baseline and year 10 , respectively, were $80.4(75.8,85.0)$ and $79.1(74.1,84.0)$ in females and $95.0(88.8,101.2)$ and 77. 5 (70.1, 84.9) in males (Figure 2). Stratification by baseline renal function (Figure 3) showed that patients with eGFR $\geq 60$ $\mathrm{mL} / \mathrm{min} / 1.73 \mathrm{~m}^{2}$ at baseline had a relatively stable mean $(95 \%$ CI) eGFR slope $\left(\mathrm{mL} / \mathrm{min} / 1.73 \mathrm{~m}^{2} /\right.$ year $)$ of $-0.55(-1.12$, $+0.01)$ for females $(n=52)$ throughout the 10-year treatment period and a small negative slope for males $(n=79)$ of -1.99 $(-2.45,-1.54)$. Among those with impaired kidney function $\left(\mathrm{eGFR}<60 \mathrm{~mL} / \mathrm{min} / 1.73 \mathrm{~m}^{2}\right)$ at baseline, the mean $(95 \% \mathrm{CI})$ eGFR slope $\left(\mathrm{mL} / \mathrm{min} / 1.73 \mathrm{~m}^{2} /\right.$ year $)$ throughout the 10 -year treatment period for females $(\mathrm{n}=10)$ was stable at $-0.14(-1.43$, $+1.15)$ and showed a modest decline for males $(n=11),-2.79$ $(-4.01,-1.56)$. For the renal cohort $(n=152)$, blood pressure values were available for 136 patients at baseline; however, the follow-up data for blood pressure were missing for many patients meaning that a blood pressure change could not be adequately analyzed.

\section{Evaluable Treated Cardiac Cohort}

The evaluable treated cardiac cohort consisted of 69 patients: 34 females with a median (range) age at treatment start of

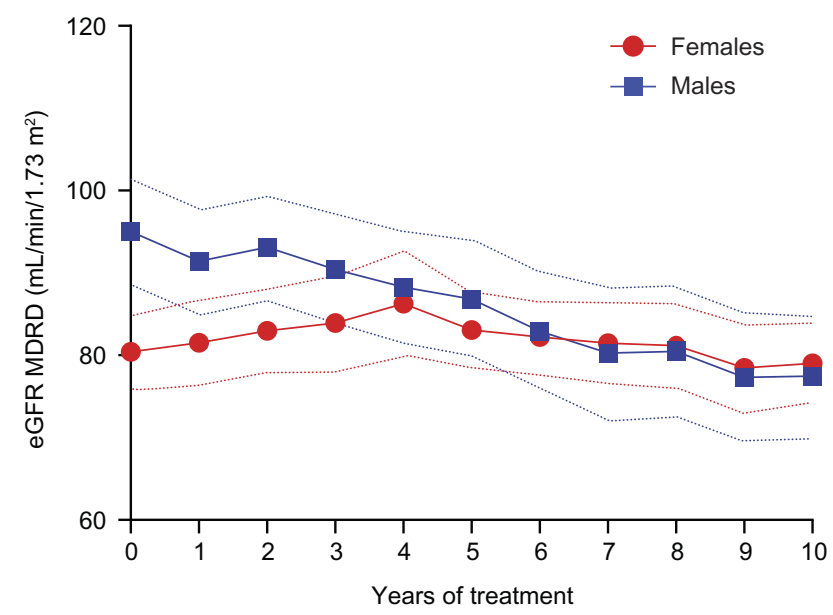

\begin{tabular}{|l|c|c|c|c|c|c|c|c|c|c|c|}
\hline Treatment year & 0 & 1 & 2 & 3 & 4 & 5 & 6 & 7 & 8 & 9 & 10 \\
\hline Females, $\mathrm{n}$ & 62 & 58 & 53 & 56 & 51 & 53 & 54 & 52 & 49 & 54 & 62 \\
\hline Males, $\mathrm{n}$ & 90 & 80 & 83 & 78 & 81 & 77 & 83 & 72 & 78 & 71 & 90 \\
\hline
\end{tabular}

Figure 2 Mean $(95 \% \mathrm{Cl})$ eGFR MDRD $\left(\mathrm{mL} / \mathrm{min} / \mathrm{l} .73 \mathrm{~m}^{2}\right)$ over a 10 -year duration of enzyme replacement therapy for female and male populations in the evaluable treated renal cohort*. *Data shown are cross-sectional values.

Abbreviations: eGFR, estimated glomerular filtration rate; MDRD, Modification of Diet in Renal Disease.
46.7 (3.7-67.3) years and 35 males with a median (range) age at treatment start of $28.2(4.0-54.2)$ years (Table 1). The median (range) time from treatment start to data extraction from FOS was 12.8 (10.1-16.5) years in females and 14.3 (10.2-17.1) years in males (Table 1). Baseline LVH was present in $18(52.9 \%)$ females and $14(40.0 \%)$ males (Table 1).

The mean $(95 \% \mathrm{CI})$ LVMI measurements $\left(\mathrm{g} / \mathrm{m}^{2.7}\right)$ at baseline and year 10, respectively, were $52.2(46.3,58.1)$ and $58.8(48.7,68.9)$ in females and $49.1(43.3,54.9)$ and $52.0(43.2,60.8)$ in males (Figure 4).

Stratification by the presence or absence of LVH at baseline (Figure 5) showed that in patients without LVH at baseline, cardiac mass remained stable in females and in males; the mean $(95 \% \mathrm{CI})$ LVMI slopes $\left(\mathrm{g} / \mathrm{m}^{2.7} /\right.$ year) throughout the 10 -year treatment period were $+0.52(-0.13,+1.17)$ for females $(n=16)$ and $+0.57(+0.02,+1.13)$ for males $(n=21)$. Among those with LVH at baseline, mean (95\% CI) LVMI $\left(\mathrm{g} / \mathrm{m}^{2.7} /\right.$ year) had slight positive slopes throughout the 10year treatment period of $+1.51(+0.91,+2.12)$ for females $(n=18)$ and $+0.87(+0.19,+1.55)$ for males $(n=14)$.

Stratification by baseline eGFR (Figure 6) showed that in patients with baseline eGFR $<60 \mathrm{~mL} / \mathrm{min} / 1.73 \mathrm{~m}^{2}$, the mean $(95 \% \mathrm{CI})$ slope (mm/year) of PWTD over the 10 year treatment period was $+0.01(-0.12,+0.15)$ for females $(n=6)$ and $+0.03(-0.11,+0.18)$ for males $(n=4)$. Among those with baseline eGFR $\geq 60 \mathrm{~mL} / \mathrm{min} / 1.73 \mathrm{~m}^{2}$, the mean $(95 \% \mathrm{CI})$ slope was $+0.10(+0.01,+0.18)$ for females $(n=24)$ and $+0.11(+0.03,+0.19)$ for males $(n=23)$.

Among cardiac cohort patients without renal involvement (ie, eGFR $>90 \mathrm{~mL} / \mathrm{min} / 1.73 \mathrm{~m}^{2}$ and urinary protein $<0.5 \mathrm{~g} /$ day) at baseline (6 of 34 [17.6\%] females and 10 of 35 [28.6\%] males), the mean (95\% CI) LVMI measurements $\left(\mathrm{g} / \mathrm{m}^{2.7}\right)$ at baseline and year 10 , respectively, were 43.3 $(27.1,59.5)$ and $45.9(22.9,69.0)$ in females $(\mathrm{n}=6)$ and 39.3 $(34.1,44.5)$ and $43.2(36.3,50.1)$ in males $(n=10$; Figure 7$)$.

\section{Discussion}

Our current analysis of a subset of the FOS population showed that during 10 years of agalsidase alfa ERT, renal function was relatively stable in females and showed modest decreases in males; these observations should be considered in the context of the natural history of Fabry disease as described in the literature. Schiffmann et al ${ }^{14}$ published a retrospective chart review of 168 female and 279 male untreated patients examined between 1944 and 2002 in North America and Europe, with only approximately one- 
A

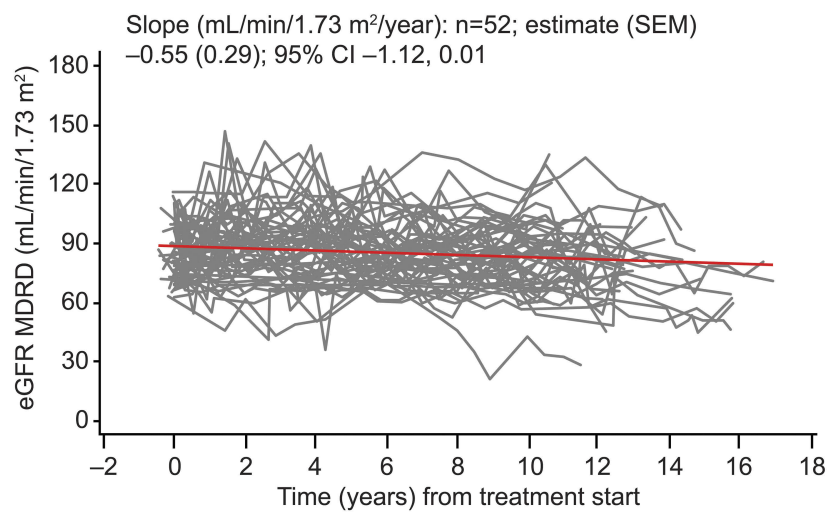

C

Slope $\left(\mathrm{mL} / \mathrm{min} / 1.73 \mathrm{~m}^{2} /\right.$ year): $\mathrm{n}=79$; estimate (SEM)

$-1.99(0.23) ; 95 \% \mathrm{Cl}-2.45,-1.54$

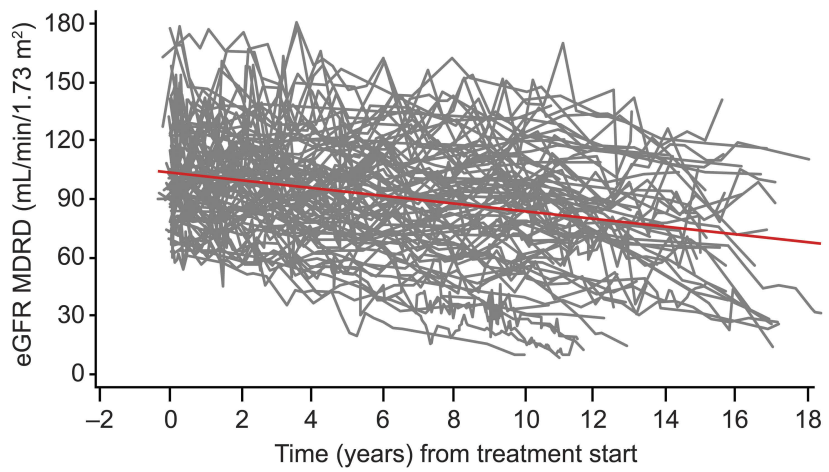

B

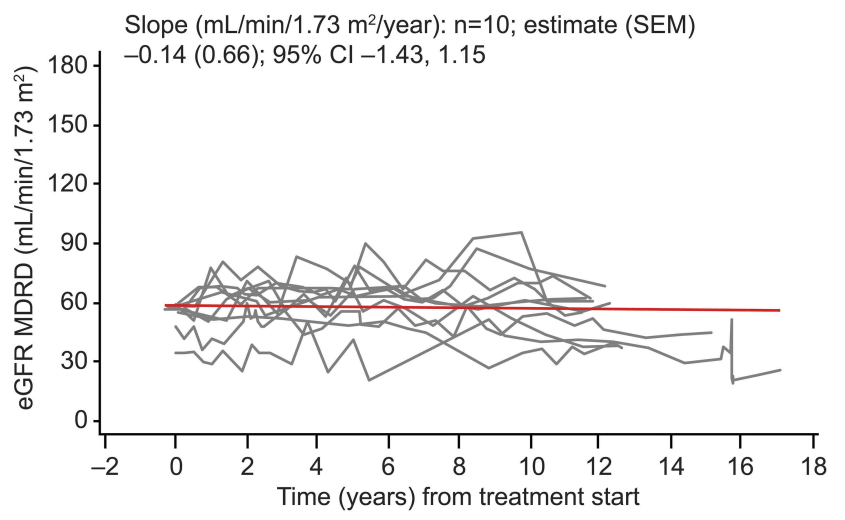

D

Slope (mL/min $1.73 \mathrm{~m}^{2} /$ year): $\mathrm{n}=11$; estimate (SEM) -2.79 (0.62); $95 \% \mathrm{Cl}-4.01,-1.56$

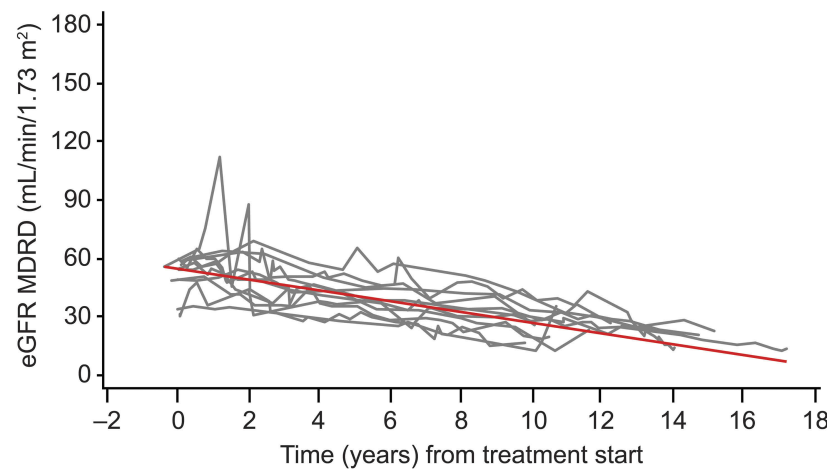

Figure 3 Individual profiles and average slope estimates for eGFR over time for the evaluable renal cohort $(n=152)$ for the: (A) female population, baseline eGFR $\geq 60 \mathrm{~mL} /$ $\mathrm{min} / 1.73 \mathrm{~m}^{2}$; (B) female population, baseline eGFR $<60 \mathrm{~mL} / \mathrm{min} / 1.73 \mathrm{~m}^{2}$; (C) male population, baseline eGFR $\geq 60 \mathrm{~mL} / \mathrm{min} / 1.73 \mathrm{~m}$; (D) male population, baseline eGFR <60 $\mathrm{mL} / \mathrm{min} / 1.73 \mathrm{~m}^{2}$. Full interaction model: [age] $\times[\mathrm{sex}] \times[$ baseline] category.

Abbreviations: eGFR, estimated glomerular filtration rate; MDRD, Modification of Diet in Renal Disease; SEM, standard error of the mean.

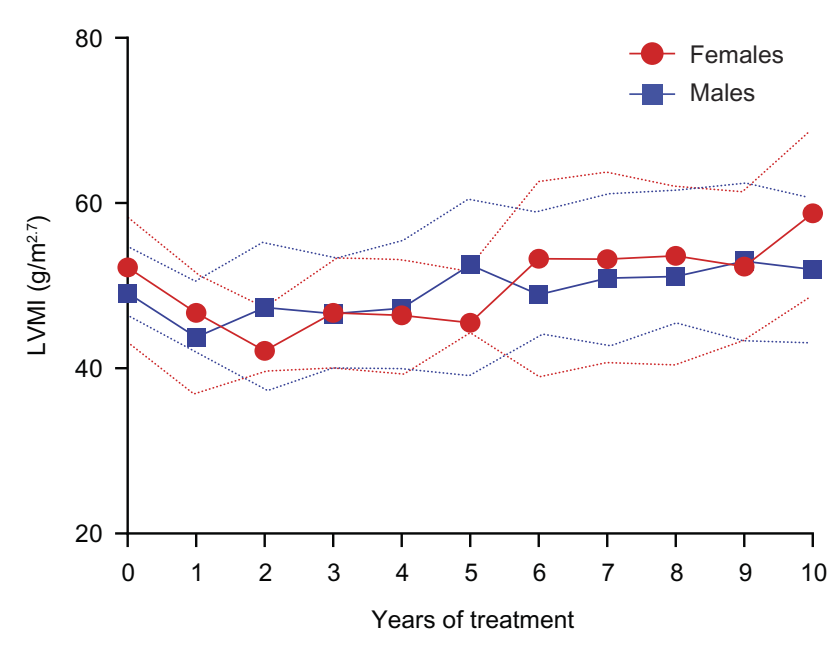

\begin{tabular}{|l|c|c|c|c|c|c|c|c|c|c|c|}
\hline Treatment year & 0 & 1 & 2 & 3 & 4 & 5 & 6 & 7 & 8 & 9 & 10 \\
\hline Females, $\mathrm{n}$ & 34 & 29 & 22 & 25 & 26 & 23 & 23 & 21 & 23 & 19 & 34 \\
\hline Males, $\mathrm{n}$ & 35 & 30 & 29 & 29 & 29 & 26 & 25 & 24 & 24 & 25 & 35 \\
\hline
\end{tabular}

Figure 4 Mean $(95 \% \mathrm{Cl}) \mathrm{LVMI}\left(\mathrm{g} / \mathrm{m}^{2.7}\right)$ over a 10 -year duration of enzyme replacement therapy for female and male populations in the evaluable treated cardiac cohort*. *Data shown are cross-sectional values.

Abbreviations: LVMI, left ventricular mass index. third of these patients receiving angiotensin receptor blockers or angiotensin-converting enzyme inhibitors. Among those with baseline eGFR $<60 \mathrm{~mL} / \mathrm{min} / 1.73 \mathrm{~m}^{2}$ (calculated from serum creatinine measurements with the abbreviated Modification of Diet in Renal Disease [MDRD] equation), the mean eGFR change was -2.1 and $-6.8 \mathrm{~mL} / \mathrm{min} / 1.73$ $\mathrm{m}^{2} /$ year, respectively, in females and males. Among those with baseline eGFR $\geq 60 \mathrm{~mL} / \mathrm{min} / 1.73 \mathrm{~m}^{2}$, the mean eGFR change was -0.9 and $-3.0 \mathrm{~mL} / \mathrm{min} / 1.73 \mathrm{~m}^{2} /$ year, respectively, in females and males. Male patients and those with hypertension, baseline proteinuria, or impaired renal function at baseline had a more rapid loss of renal function.

A FOS analysis by Mehta et al of adults who received 5 years of agalsidase alfa showed mean annual eGFR changes of -0.89 and $-3.17 \mathrm{~mL} / \mathrm{min} / 1.73 \mathrm{~m}^{2}$ in females and males, respectively. ${ }^{15}$ The authors noted these declines in eGFR (calculated from serum creatinine measurements with the MDRD equation) were slower than those reported for the natural history of the disease and were similar to those 
A

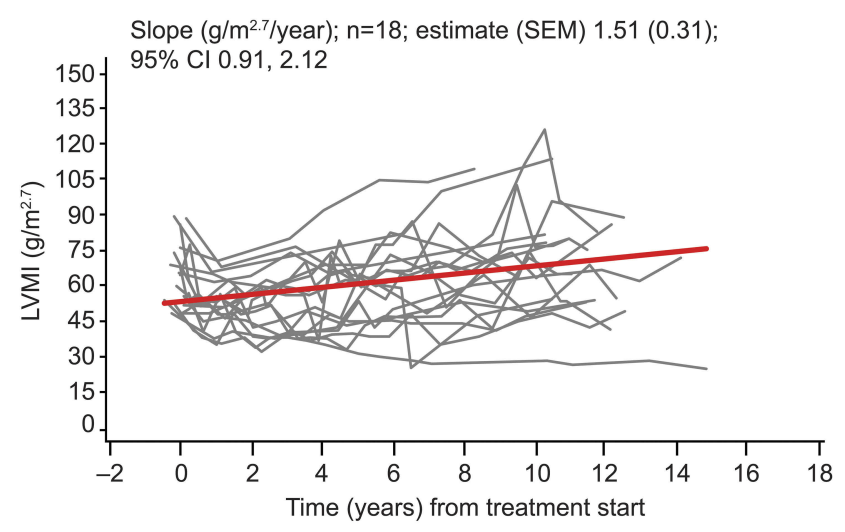

C

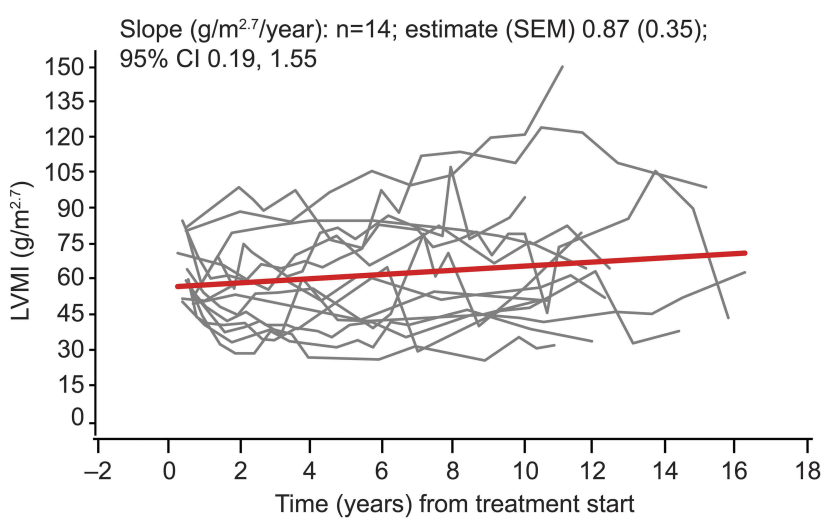

B

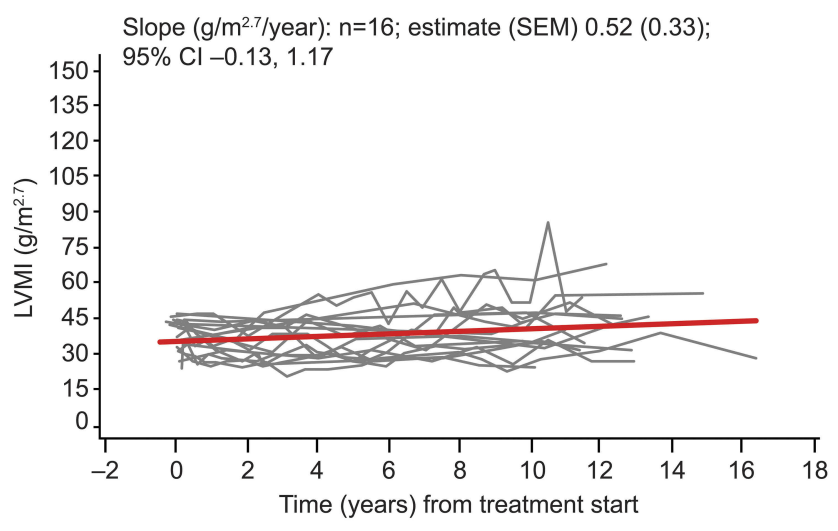

D

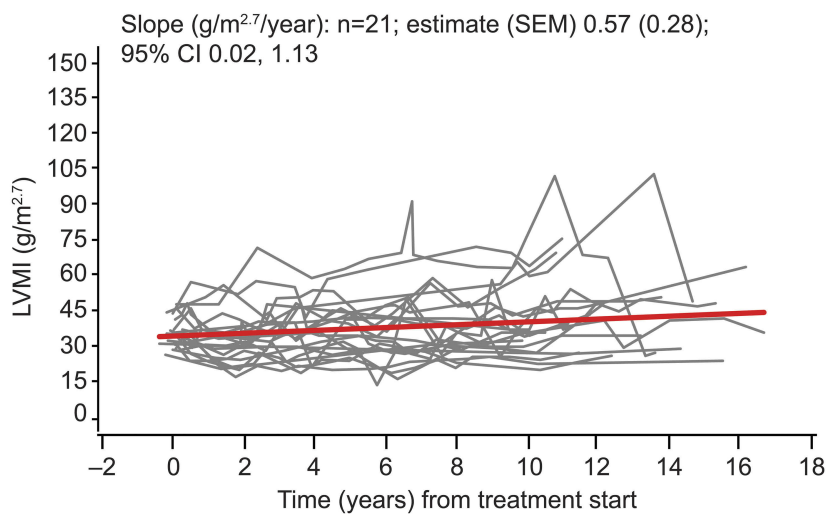

Figure 5 Individual profiles and average slope estimates for LVMI over time for the evaluable cardiac cohort $(n=69)$ for the $(\mathbf{A})$ female population, LVH at baseline; (B) female population, no LVH at baseline; (C) male population, LVH at baseline; (D) male population, no LVH at baseline.

Abbreviations: LVH, left ventricular hypertrophy; LVMI, left ventricular mass index; SEM, standard error of the mean.

expected with age in females and approximately 2 to 3 times greater than those expected with age in males. Among the patients with baseline eGFR $\geq 130 \mathrm{~mL} / \mathrm{min} /$ $1.73 \mathrm{~m}^{2}, \sim 86 \%$ had eGFR within the normal range after 5 years of ERT, and among patients with baseline eGFR classified as chronic kidney disease stages 1 to 3 , mean annual changes in eGFR were between -1.53 and -2.63 $\mathrm{mL} / \mathrm{min} / 1.73 \mathrm{~m}^{2}$, with renal function stabilization or improvement in $56.5 \%$ to $75.0 \%$ of the patients, and slowed decline of eGFR in $68.8 \%$ to $89.1 \%$.

Nowak et al recently published results from prospective disease progression modeling of renal function in adults with classical Fabry disease. ${ }^{16}$ During a median follow-up of 9 years in 21 treated females and 24 treated males (most received agalsidase alfa), female patients had a stable renal function (eGFR slope, $-0.07 \mathrm{~mL} / \mathrm{min} / 1.73$ $\mathrm{m}^{2} /$ year) and males had a change in eGFR of $-3.07 \mathrm{~mL} /$ $\mathrm{min} / 1.73 \mathrm{~m}^{2} /$ year. There was no clear effect of angiotensin receptor blockers or angiotensin-converting enzyme inhibitors on the eGFR slope, but there was an effect on proteinuria. The effect of these medications on proteinuria has been described in patients with various proteinuric conditions; ${ }^{17,18}$ this is also expected in Fabry disease. The effects of these medications on eGFR slope is less consistent in the literature and may be related to proteinuria reduction rather than a direct effect of the medications. The patients in the Nowak report had a median baseline eGFR of $92 \mathrm{~mL} / \mathrm{min} / 1.73 \mathrm{~m}^{2}$ in females and 96 $\mathrm{mL} / \mathrm{min} / 1.73 \mathrm{~m}^{2}$ in males (calculated using the serum creatinine-based Chronic Kidney Disease-Epidemiology Collaboration [CKD-EPI(cr)] equation); ${ }^{16}$ our FOS evaluable treated renal cohort had a mean baseline eGFR of $80.4 \mathrm{~mL} / \mathrm{min} / 1.73 \mathrm{~m}^{2}$ in females and $95.0 \mathrm{~mL} / \mathrm{min} / 1.73 \mathrm{~m}^{2}$ in males.

Germain et al published a 10-year analysis of patients receiving agalsidase beta ERT stratified by baseline renal involvement. ${ }^{3}$ The study included data collected from 50 males and 2 females enrolled in a phase 3 clinical trial and an open-label extension study, as well as subsequent data after the patients entered the Fabry Registry. Overall, 

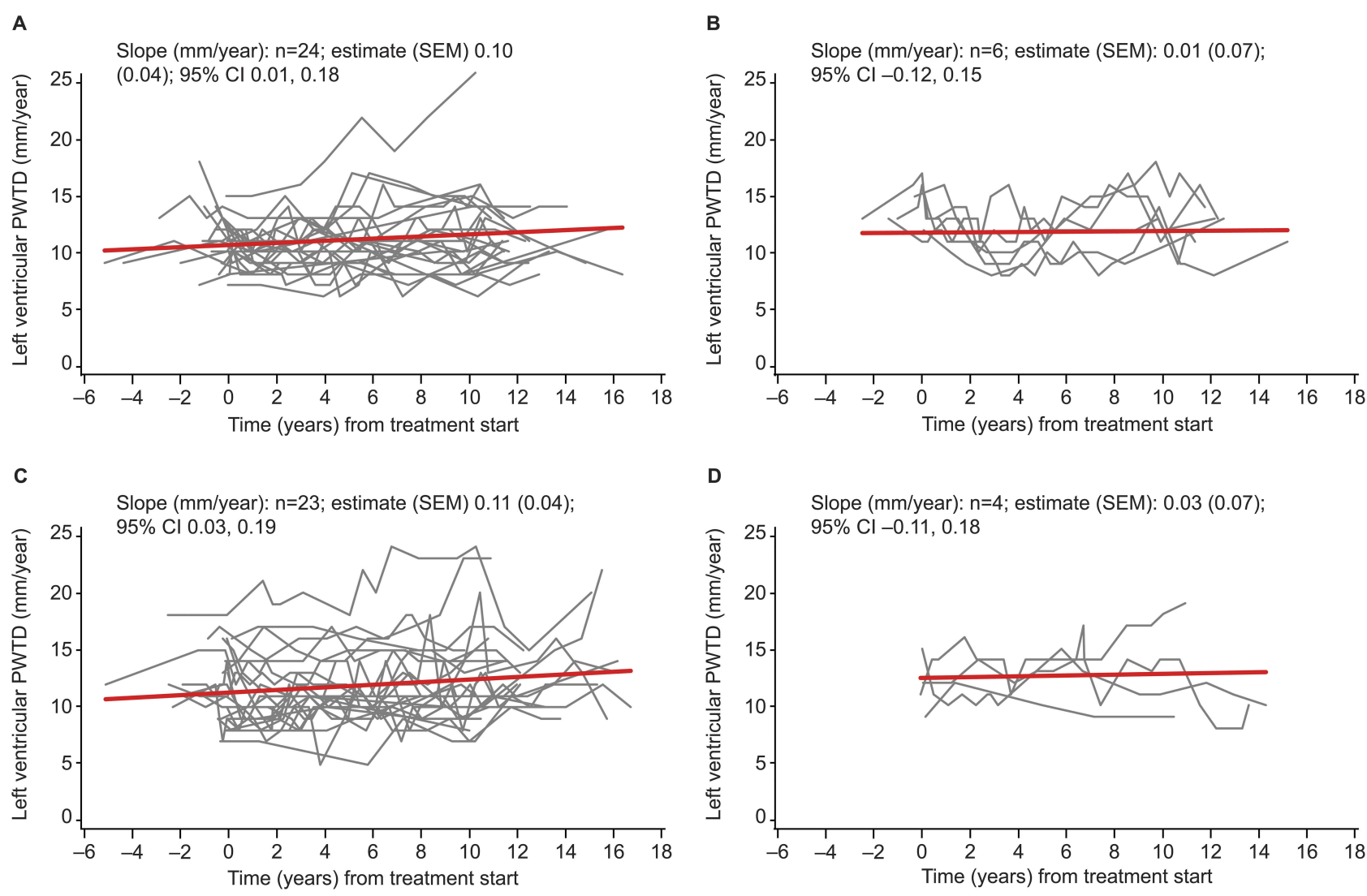

Figure 6 Individual profiles and average slope estimates for left ventricular PWTD over time for the evaluable cardiac cohort ( $\mathrm{n}=69)$ for the $(\mathbf{A})$ female population, baseline eGFR $\geq 60 \mathrm{~mL} / \mathrm{min} / 1.73 \mathrm{~m}^{2}$; (B) female population, baseline eGFR $<60 \mathrm{~mL} / \mathrm{min} / 1.73 \mathrm{~m}^{2}$; (C) male population, baseline eGFR $\geq 60 \mathrm{~mL} / \mathrm{min} / 1.73 \mathrm{~m}$; (D) $\mathrm{male}$ population, baseline eGFR $<60 \mathrm{~mL} / \mathrm{min} / 1.73 \mathrm{~m}^{2}$.

Abbreviations: eGFR, estimated glomerular filtration rate; PWTD, left ventricular posterior wall thickness in diastole; SEM, standard error of the mean.

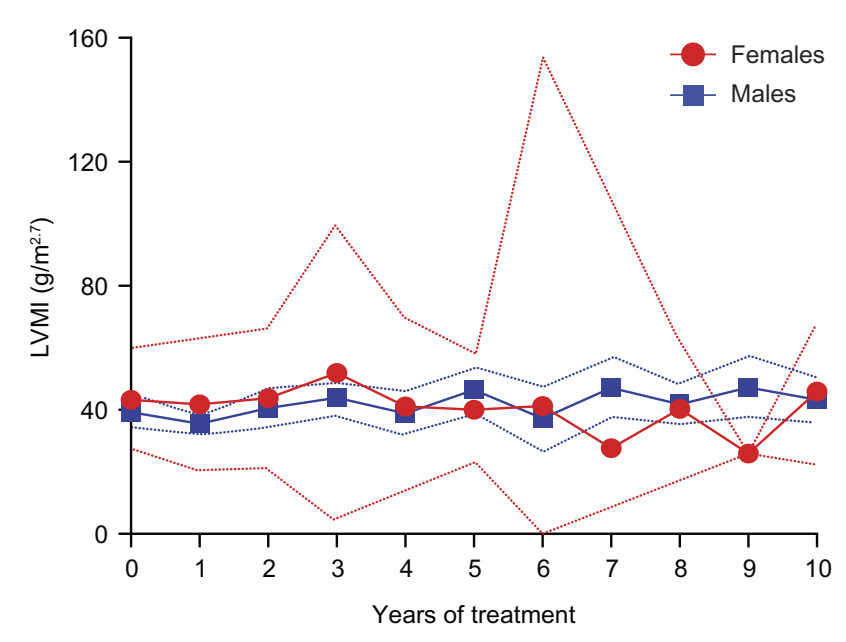

\begin{tabular}{|l|c|c|c|c|c|c|c|c|c|c|c|}
\hline Treatment year & 0 & 1 & 2 & 3 & 4 & 5 & 6 & 7 & 8 & 9 & 10 \\
\hline Females, $\mathrm{n}$ & 6 & 4 & 4 & 3 & 4 & 4 & 2 & 1 & 4 & 2 & 6 \\
\hline Males, $\mathrm{n}$ & 10 & 8 & 9 & 9 & 8 & 7 & 7 & 7 & 8 & 8 & 10 \\
\hline
\end{tabular}

Figure 7 Mean $(95 \% \mathrm{Cl}) \mathrm{LVMI}\left(\mathrm{g} / \mathrm{m}^{2.7}\right)$ over a 10 -year duration of enzyme replacement therapy for female and male patients with baseline eGFR $>90 \mathrm{~mL} / \mathrm{min} / 1.73 \mathrm{~m}^{2}$ and urinary protein $<0.5 \mathrm{~g} /$ day in the evaluable treated cardiac cohort.

Abbreviations: eGFR, estimated glomerular filtration rate; LVMI, left ventricular mass index. patients in the Germain et al study were younger at treatment start (mean age, 30.1 years) than those in our FOS evaluable renal cohort (mean age, 40.2 years), and had a higher eGFR (calculated using the serum creatinine-based CKD-EPI(cr) equation) at baseline (mean, $116.6 \mathrm{~mL} / \mathrm{min} /$ $1.73 \mathrm{~m}^{2}$ ) than patients in our FOS evaluable renal cohort (mean, $89.0 \mathrm{~mL} / \mathrm{min} / 1.73 \mathrm{~m}^{2}$ ). Germain et al classified patients as having low or high baseline renal involvement: low renal involvement was defined by a urinary protein-tocreatinine ratio of $\leq 0.5 \mathrm{~g} / \mathrm{g}$ and $<50 \%$ sclerotic glomeruli in renal biopsies collected during the first 6 months of the study, while high renal involvement was defined by a urinary protein-to-creatinine ratio of $>0.5 \mathrm{~g} / \mathrm{g}$ or $\geq 50 \%$ sclerotic glomeruli. The mean eGFR slope was -1.89 and $-6.82 \mathrm{~mL} / \mathrm{min} / 1.73 \mathrm{~m}^{2} /$ year over 10 years among patients classified as having low $(n=32)$ or high $(n=20)$ renal involvement, respectively, at baseline. We stratified our FOS evaluable renal cohort by baseline eGFR $\geq 60$ or $<60 \mathrm{~mL} / \mathrm{min} / 1.73 \mathrm{~m}^{2}$. In our analyses of those with higher baseline eGFR, renal function was relatively stable (slope 
estimate, -0.55) in 52 females and showed modest declines (slope estimate, -1.99) in 79 males over 10 years of ERT; our analysis of those with impaired renal function at baseline showed a steady eGFR (slope estimate, -0.14) in 10 females and a modest decline (slope estimate, -2.79) in 11 males. Taken together, patients treated with agalsidase alfa or beta over 10 years show better preservation of renal function when ERT was started with earlier kidney disease characterized by higher baseline eGFR and less proteinuria.

Our current FOS analysis showed that during 10 years of agalsidase alfa ERT, LVMI had relatively small increases among patients with LVH at baseline and was stable among patients without LVH at baseline. The observed increases in LVMI were less than those reported in an untreated population, in which LVMI was shown to increase at a rate of $2.31 \mathrm{~g} / \mathrm{m}^{2.7} /$ year in females and $4.07 \mathrm{~g} / \mathrm{m}^{2.7} /$ year in males. ${ }^{13}$ In addition, patients without renal involvement and $\mathrm{LVH}$ at baseline who received agalsidase alfa ERT over 10 years in FOS had stable cardio-renal outcomes, which would be beneficial in preventing early organ manifestation of the disease. In a 10year analysis by Kampmann et al of patients receiving agalsidase alfa at a single FOS site, ${ }^{19}$ males with baseline LVMI $\geq 50 \mathrm{~g} / \mathrm{m}^{2.7}$ had statistically significant reductions in LVMI from baseline at years 1, 2, 3, 5, and 10, while females had similar improvements in LVMI at years 1, 2, and 3. Reflecting our findings, in patients with baseline LVMI $<50 \mathrm{~g} / \mathrm{m}^{2.7}$, LVMI was not significantly changed from baseline over 10 years of ERT. ${ }^{19}$ The patient population, management, and follow-up in our current FOS 10 -year multicenter analysis were less homogeneous than in the single-center report by Kampmann et al, but our overall treatment outcomes were similar and appear consistent with the single-center experience.

Our current analysis also showed that over 10 years of ERT, in patients with minimal renal involvement at baseline, LVMI was stable over 10 years, between 43.3 and $45.9 \mathrm{~g} / \mathrm{m}^{2.7}$ in females and between 39.3 and $43.2 \mathrm{~g} / \mathrm{m}^{2.7}$ in males. This finding is in agreement with the results reported by Germain et al, in which the mean slopes of left posterior wall thickness over 10 years of agalsidase beta ERT were $+0.08 \mathrm{~mm} /$ year and $+0.10 \mathrm{~mm} /$ year in patients with low and high Fabry disease renal involvement, respectively, at baseline. ${ }^{3}$ Compared with the population in the Germain et al report, our cohort had similar or greater overall disease severity at baseline.
Several limitations of our study should be considered. Observational studies, including analysis of patient registry data, provide important real-world evidence that complements the limited information available from randomized controlled trials in rare diseases. However, with registry data in general, it is difficult to ensure comprehensive data capture, assessments may not be standardized across multiple sites, and there is the possibility of patient enrollment bias as patients with more severe symptoms or who are receiving treatment are more likely to be enrolled in a registry. This retrospective analysis of 10-year FOS patient registry data did not include comparisons with untreated patients from the same registry because there were too few untreated patients to match by sex, age, and/or disease severity. The patients in the FOS registry were not randomly selected, possibly giving rise to selection and/or ascertainment bias. For example, male patients may have been identified and treated earlier than females, whereas treatment start may have in some cases been delayed in females until organ involvement was established (as suggested by lower eGFR at baseline). Although the serum creatinine-based CKD-EPI(cr) equation may provide a more accurate estimation at eGFR $>60 \mathrm{~mL} / \mathrm{min} / 1.73 \mathrm{~m}^{2}$, the MDRD equation is widely used, ${ }^{20}$ allowing comparison with more studies, and was used in the FOS 5-year analysis. However, proteinuria and its effect on eGFR slopes were also not fully investigated in this analysis. There was no centralized reading of echocardiograms in the FOS cohorts. There is the possibility of bias associated with the censoring of patients who stopped agalsidase alfa treatment. The relatively small sample size, especially in subgroups, could affect the precision and robustness of estimates, and the lack of data regarding Fabry disease phenotype, proteinuria, and blood pressure control may limit the generalizability of our observations. The relationship of the surrogate endpoints in this analysis to clinical endpoints is not clear. By the nature of the analysis, the clinical endpoints may be enriched in people who died or stopped ERT.

\section{Conclusion}

The current analysis of a cohort of FOS provides evidence supporting the benefits of agalsidase alfa ERT over 10 years. Patients with preserved kidney function and/or normal LVMI at baseline remained largely stable during 10 years of ERT, whereas those patients with reduced eGFR and/or evidence of LVH at baseline experienced modest declines in renal function and/or increases in LVMI that were less than expected based on natural history data. These findings suggest that early treatment, before substantial organ damage has occurred, may improve outcomes. 


\section{Acknowledgments}

Under the direction of the authors, Margit Rezabek, DVM, $\mathrm{PhD}$, Sally Hassan, PhD, CMPP, and Latoya M. Mitchell, PhD, CMPP, employees of Excel Medical Affairs, provided writing assistance for this publication. Editorial assistance in formatting, proofreading, copy editing, and fact checking was also provided by Excel Medical Affairs. Statistical support was provided by Patrick Engrand, PhD, of Clinical Research Services - ICC, under contract to Shire. This paper was presented at the WORLDSymposium 2016 as a poster presentation with interim findings. The poster's abstract was published in Ramaswami U, et al Mol Genet Metab 2016;117(2):S98. https://doi.org/10.1016/j.ymgme. 2015.12.416.

\section{Funding}

The Fabry Outcome Survey is sponsored by Shire Human Genetic Therapies, Inc., a member of the Takeda group of companies. Shire International $\mathrm{GmbH}$, a member of the Takeda group of companies, provided funding to Excel Medical Affairs for support in writing and editing this manuscript and participated in data collection and analysis.

\section{Disclosure}

Uma Ramaswami has received travel and research grants and honoraria for lectures and advisory boards from Amicus, Chiesi, Genzyme, and Shire (now a member of the Takeda group of companies). She is also a member of the steering committee (SC) of FOS, a registry of Fabry disease sponsored by Shire/Takeda, and has received honoraria for compensation of the time spent on SC meetings. Michael Beck has received consulting fees from Genzyme and Shire. He is also a member of the SC of FOS and has received honoraria for compensation of the time spent on SC meetings. Derralynn Hughes has received travel and research grants and honoraria for speaking and advisory boards from Amicus, Genzyme, Protalix, and Shire. She also reports personal fees from Takeda, Sanofi, and Amicus, outside the submitted work. She is also a member of the SC of FOS and has received honoraria for compensation of the time spent on $\mathrm{SC}$ meetings. Christoph Kampmann has received consulting fees from Amicus, BioMarin, Gore, and Shire and honoraria for lectures and travel and research grants from BioMarin, Shire, and Gore. He is also a member of the SC of FOS and has received honoraria for compensation of the time spent on $\mathrm{SC}$ meetings. Jaco Botha is an employee of Takeda and a Takeda stock owner. Guillem Pintos-Morell has received consulting fees, honoraria for lectures, and travel grants from Alexion, Amicus, BioMarin, and Shire, during the conduct of the study. He is also a member of the SC of FOS and has received honoraria for compensation of the time spent on SC meetings. Michael West has received consulting fees from GlaxoSmithKline, AvroBio and Shire; has performed contract research for Alexion, Genzyme, Idorsia and Shire; has received speaker honoraria from Excelsior, Genzyme, Shire, and Sumitomo; honoraria for serving on a data and safety monitoring board for Amicus; and honoraria for advisory boards from Actelion, Genzyme, and Shire. He is also a member of the SC of FOS and has received honoraria for compensation of the time spent on SC meetings. DauMing Niu has received travel and research grants and speaker honoraria from Genzyme and Shire. He is also a member of the SC of FOS and has received honoraria for compensation of the time spent on SC meetings. Kathy Nicholls reports grants from Melbourne Health, during the conduct of the study and has received research support from Amicus, Genzyme, and Shire, outside the submitted work. She is also a member of the $\mathrm{SC}$ of $\mathrm{FOS}$ and has received honoraria for compensation of the time spent on SC meetings. Roberto Giugliani has received consulting fees, fees for non-CME/CE services, investigator fees, and support for travel expenses to attend scientific meetings from Actelion, Amicus, Armagen, BioMarin, GC Pharma, Inventiva, JCR Pharmaceuticals, Lysogene, RegenxBio, Sanofi-Genzyme, Shire, Sobi and Ultragenyx. He is also a member of the SC of FOS and has received honoraria for compensation of the time spent on SC meetings. The authors report no other conflicts of interest in this work.

\section{References}

1. Germain DP. Fabry disease. Orphanet J Rare Dis. 2010;5:30. doi:10.1186/1750-1172-5-30

2. Ramaswami U, Wendt S, Pintos-Morell G, et al. Enzyme replacement therapy with agalsidase alfa in children with Fabry disease. Acta Paediatr. 2007;96(1):122-127. doi:10.1111/j.1651-2227.2007.00029.x

3. Germain DP, Charrow J, Desnick RJ, et al. Ten-year outcome of enzyme replacement therapy with agalsidase beta in patients with Fabry disease. J Med Genet. 2015;52(5):353-358. doi:10.1136/jmedgenet-2014-102797

4. Hughes DA. Fabry disease: will markers of early disease enable early treatment and better outcomes? Curr Opin Cardiol. 2016;31(4):434439. doi:10.1097/HCO.0000000000000308

5. Ortiz A, Abiose A, Bichet DG, et al. Time to treatment benefit for adult patients with Fabry disease receiving agalsidase beta: data from the Fabry Registry. J Med Genet. 2016;53(7):495-502. doi:10.1136/ jmedgenet-2015-103486

6. Weidemann F, Niemann M, Breunig F, et al. Long-term effects of enzyme replacement therapy on Fabry cardiomyopathy: evidence for a better outcome with early treatment. Circulation. 2009;119(4):524529. doi:10.1161/CIRCULATIONAHA.108.794529 
7. Oder D, Nordbeck P, Wanner C. Long term treatment with enzyme replacement therapy in patients with Fabry disease. Nephron. 2016;134(1):30-36. doi:10.1159/000448968

8. Giugliani R, Niu DM, Ramaswami U, et al. A 15-year perspective of the Fabry Outcome Survey. J Inborn Errors Metab Screen. 2016;4:112. doi:10.1177/2326409816666298

9. Beck M, Hughes D, Kampmann C, et al. Long-term effectiveness of agalsidase alfa enzyme replacement in Fabry disease: a Fabry Outcome Survey analysis. Mol Genet Metab Rep. 2015;3:21-27. doi:10.1016/j.ymgmr.2015.02.002

10. Levey AS, Coresh J, Bolton K, et al. K/DOQI clinical practice guidelines for chronic kidney disease: evaluation, classification, and stratification. Am J Kidney Dis. 2002;39(2 Suppl 1):S1-266.

11. Linhart A, Kampmann C, Zamorano JL, et al. Cardiac manifestations of Anderson-Fabry disease: results from the international Fabry Outcome Survey. Eur Heart J. 2007;28(10):1228-1235. doi:10.1093/eurheartj/ ehm153

12. de Simone G, Devereux RB, Daniels SR, Koren MJ, Meyer RA, Laragh JH. Effect of growth on variability of left ventricular mass: assessment of allometric signals in adults and children and their capacity to predict cardiovascular risk. J Am Coll Cardiol. 1995;25 (5):1056-1062. doi:10.1016/0735-1097(94)00540-7

13. Kampmann C, Linhart A, Baehner F, et al. Onset and progression of the Anderson-Fabry disease related cardiomyopathy. Int J Cardiol. 2008;130(3):367-373. doi:10.1016/j.ijcard.2008.03.007

14. Schiffmann R, Warnock DG, Banikazemi M, et al. Fabry disease: progression of nephropathy, and prevalence of cardiac and cerebrovascular events before enzyme replacement therapy. Nephrol Dial Transplant. 2009;24(7):2102-2111. doi:10.1093/ndt/gfp031
15. Mehta A, Beck M, Elliott P, et al. Enzyme replacement therapy with agalsidase alfa in patients with Fabry's disease: an analysis of registry data. Lancet. 2009;374(9706):1986-1996. doi:10.1016/S0140-6736(09) 61493-8

16. Nowak A, Koch G, Huynh-Do U, Siegenthaler M, Marti HP, Pfister M. Disease progression modeling to evaluate the effects of enzyme replacement therapy on kidney function in adult patients with the classic phenotype of Fabry disease. Kidney Blood Press Res. 2017;42 (1):1-15. doi:10.1159/000464312

17. Kunz R, Friedrich C, Wolbers M, Mann JF. Meta-analysis: effect of monotherapy and combination therapy with inhibitors of the renin angiotensin system on proteinuria in renal disease. Ann Intern Med. 2008;148(1):30-48. doi:10.7326/0003-4819-148-1-200801010-00190

18. Xu R, Sun S, Huo Y, et al. Effects of ACEIs versus ARBs on proteinuria or albuminuria in primary hypertension: a meta-analysis of randomized trials. Medicine (Baltimore). 2015;94(39):e1560. doi:10.1097/MD.0000000000000874

19. Kampmann C, Perrin A, Beck M. Effectiveness of agalsidase alfa enzyme replacement in Fabry disease: cardiac outcomes after 10 years' treatment. Orphanet J Rare Dis. 2015;10:125. doi:10.1186/s13023-015-0338-2

20. Kilbride HS, Stevens PE, Eaglestone G, et al. Accuracy of the MDRD (Modification of Diet in Renal Disease) study and CKDEPI (CKD Epidemiology Collaboration) equations for estimation of GFR in the elderly. Am J Kidney Dis. 2013;61(1):57-66. doi:10.1053/ j.ajkd.2012.06.016
Drug Design, Development and Therapy

\section{Publish your work in this journal}

Drug Design, Development and Therapy is an international, peerreviewed open-access journal that spans the spectrum of drug design and development through to clinical applications. Clinical outcomes, patient safety, and programs for the development and effective, safe, and sustained use of medicines are a feature of the journal, which has also

\section{Dovepress}

been accepted for indexing on PubMed Central. The manuscript management system is completely online and includes a very quick and fair peer-review system, which is all easy to use. Visit http://www. dovepress.com/testimonials.php to read real quotes from published authors. 